\title{
Validation of a simple and inexpensive method for the quantitation of infarct in the rat brain
}

\section{C.L.R. Schilichting, K.C.M. Lima, \\ L.A. Cestari Junior, \\ and H. Milani}

J.Y. Sekiyama, F.M. Silva
Departamento de Farmácia e Farmacologia, Centro de Ciências da Saúde, Universidade Estadual de Maringá, Maringá, PR, Brasil

\section{Correspondence}

H. Milani

Departamento de Farmácia e

Farmacologia, CCS, UEM

Av. Colombo, 5790

87020-900 Maringá, PR

Brasil

Fax: + 55-44-263-6231

E-mail: hmilani@uem.br

Research supported by CNPq and UEM.

Received April 8, 2003

Accepted January 5, 2004

\section{Abstract}

A gravimetric method was evaluated as a simple, sensitive, reproducible, low-cost alternative to quantify the extent of brain infarct after occlusion of the medial cerebral artery in rats. In ether-anesthetized rats, the left medial cerebral artery was occluded for $1,1.5$ or $2 \mathrm{~h}$ by inserting a 4-0 nylon monofilament suture into the internal carotid artery. Twenty-four hours later, the brains were processed for histochemical triphenyltetrazolium chloride (TTC) staining and quantitation of the schemic infarct. In each TTC-stained brain section, the ischemic tissue was dissected with a scalpel and fixed in $10 \%$ formalin at $0^{\circ} \mathrm{C}$ until its total mass could be estimated. The mass $(\mathrm{mg})$ of the ischemic tissue was weighed on an analytical balance and compared to its volume $\left(\mathrm{mm}^{3}\right)$, estimated either by plethysmometry using platinum electrodes or by computer-assisted image analysis. Infarct size as measured by the weighing method ( $\mathrm{mg})$, and reported as a percent (\%) of the affected (left) hemisphere, correlated closely with volume $\left(\mathrm{mm}^{3}\right.$, also reported as \%) estimated by computerized image analysis $(\mathrm{r}=0.88 ; \mathrm{P}<0.001 ; \mathrm{N}=10)$ or by plethysmography $(\mathrm{r}=0.97-0.98 ; \mathrm{P}$ $<0.0001 ; \mathrm{N}=41)$. This degree of correlation was maintained between different experimenters. The method was also sensitive for detecting the effect of different ischemia durations on infarct size $(\mathrm{P}<0.005 ; \mathrm{N}$ $=23$ ), and the effect of drug treatments in reducing the extent of brain damage $(\mathrm{P}<0.005 ; \mathrm{N}=24)$. The data suggest that, in addition to being simple and low cost, the weighing method is a reliable alternative for quantifying brain infarct in animal models of stroke.

\section{Key words}

- Focal cerebral ischemia

- Infarct size

- Rats

- Triphenyltetrazolium

chloride stain

- Weighing method

\section{Introduction}

The size of infarcts has been frequently used as the primary indicator of the extent of ischemic brain damage in animal models of stroke. Triphenyltetrazolium chloride (TTC) (1) is one of the most frequently employed histochemical staining methods for reliable macroscopic identification of infarcted tissue (2-9). The principle of TTC staining relies on the oxidation of TTC by intact mitochondrial dehydrogenase, which yields the carmin red product, formazan. Infarcted (necrotic) tissues lack dehydrogenase activ- 
ity and therefore do not stain. Thus, the infarcted area (white) can be easily distinguished from the surrounding intact tissues (red) (1).

The estimation of infarct size from TTCstained coronal brain slices has been greatly facilitated by the development of semiautomatic (10) and automated (11) computerassisted image analysis systems. Although these methods have been widely accepted owing to their practicality, their cost hinders work in the field of cerebral ischemia by many laboratories. Given this difficulty, we systematically evaluated a simple and inexpensive alternative method to quantify brain infarcts in the rat.

We analyzed a method for dissecting and weighing ischemic tissue to estimate the extent of brain infarct. The technique requires an analytical laboratory balance. Provided that TTC staining of high quality can be attained, reliable dissection of the infarcted tissue is guaranteed. The method has been used previously to quantify the neuroprotective effect of barbiturates after cerebral ischemia in cats (12). However, no effort has been made to validate the technique as a sensitive, reproducible and reliable method for infarct quantification. In the present study we propose a systematic evaluation of the method of dissecting and weighing infarcted tissue as a useful tool to quantify brain infarcts in experimental animals.

\section{Material and Methods}

\section{Animals}

Adult male Wistar rats weighing 280$300 \mathrm{~g}$ were used. The animals were housed in groups of 3 to 4 in plastic cages $(39 \times 33 \times$ $16 \mathrm{~cm})$ under controlled temperature $(22 \pm$ $1^{\circ} \mathrm{C}$ ) on a 12-h light-dark cycle (lights on at 7:00 h) with constant air replacement. Food and water were offered ad libitum. These conditions were maintained throughout the experiments.

\section{Animal preparation}

Regional brain ischemia was induced by proximal occlusion of the left medial cerebral artery (MCA) according to the method described by Longa et al. (4). Briefly, rats were fasted overnight, and under ether anesthesia, the left common carotid, external carotid (ECA), internal carotid (ICA) and pterygopalatine arteries were exposed and carefully separated from adjacent tissue. Occlusion of the MCA was performed by inserting an Ethicon 4-0 nylon monofilament suture into the ICA, via the ECA, and advancing gently until a weak resistance is felt. Using this criterion, the extent of introduction of the monofilament varied from 19 to $22 \mathrm{~mm}$ from the ICA/ECA bifurcation. Once the monofilament was inserted into the ECA and advanced close to the ICA/ECA bifurcation, anesthesia was interrupted and 3 to 4 min later the filament was advanced to occlude the MCA. Prior to use, the filament tip was rounded by heating on a flame and checked microscopically (10X) for the presence of a spiked or cutting surface. Only those filaments with a smooth surface and well-rounded tip were used. Rectal temperature was monitored with a digital thermometer (Minipa, APPA MT-520, São Paulo, SP, Brazil) using a rectal probe inserted to a depth of approximately $6 \mathrm{~cm}$. Core temperature was controlled only during surgery and maintained around $37.5^{\circ} \mathrm{C}$ by a heating blanket. During ischemia and reperfusion, temperature was monitored but not controlled, except when necessary (experiment 3 ). At the end of ischemia, the animal was briefly reanesthetized and reperfusion was permitted by retracting the filament from the ICA, into the ECA. The suture was closed and the animal was killed the following day. Experimental procedures followed the ethical principles of the Brazilian College of Animal Experimentation published by the Brazilian Society for Neuroscience and Behavior. 


\section{Tissue staining}

Twenty-four hours after MCA occlusion, the animal was deeply anesthetized with ether and decapitated and the brain was removed quickly and immersed in ice-cold saline (1$2^{\circ} \mathrm{C}$ ) for $10 \mathrm{~min}$. The brain was subsequently placed in a chilled brain matrix and sectioned coronally into eight to nine $1.5-\mathrm{mm}$ thick slices starting from the frontal pole. The olfactory bulb and cerebellum were discarded. The brain was irrigated continuously with cold saline during sectioning. Each slice was then immersed in $10 \mathrm{ml}$ of $2.0 \%$ 2,3,5TTC (Sigma, St. Louis, MO, USA) for 30 min at $37^{\circ} \mathrm{C}$ (15 min with each surface facing up). After staining, the slices were washed twice in $10 \mathrm{ml}$ saline, fixed in $10 \%$ buffered formalin, $\mathrm{pH} 7.4$, and left to stand at $4^{\circ} \mathrm{C}$ overnight in a lightproof container (5).

\section{Estimation of extent of brain infarct by weighing, plethysmography and image analysis}

The TTC-stained brain sections were placed on a smooth glass surface and the infarcted necrotic tissue (white in color) was separated from the adjacent intact tissue (carmin red in color) using a \#11 microscalpel blade. A magnifying lens was unnecessary since the infarcted, necrotic tissue could be easily discerned and macroscopically manipulated at any depth within the section. After separation, the normal tissues of both the intact and the affected hemispheres, and the ischemic tissue, were transferred to Eppendorf tubes containing $10 \%$ buffered formalin and held at $0^{\circ} \mathrm{C}$ until weighing (balance) and volume measurement (plethysmography), when the tissues were transferred to a Petri dish and washed twice ( 2 to 3 min each) in $0.07 \%$ saline (plethysmometer electrolyte solution). Excess saline on the surface of the tissue was carefully blotted with a swab. The ischemic tissue was then weighed using an analytical balance (Mettler-Toledo GmbH, Model AB204-S, Greifensee, Swit- zerland) and its volume was measured using a plethysmometer coupled to platinum electrodes (Ugo Basile, 7150 Plethysmometer, ComerioVarese, Italy). In the first part of this study (see Experimental protocol: experiment 1), a semiautomated image analysis system (Image Pro Plus 4.50.29, Media Cybernetics, Inc., Silver Spring, MD, USA) was also used to estimate the infarcted area $\left(\mathrm{mm}^{2}\right)$ from each TTC-stained brain slice. Digital images were captured from the TTC-stained slices using a color scanner (Scanjet 4C, Hewlett-Packard) linked to a personal computer (Pentium 4, 1.6 $\mathrm{Gb}, 256 \mathrm{Mb}$ RAM) equipped with image processing software (Paint-Shop Pro, 7.1). The total infarct volume $\left(\mathrm{mm}^{3}\right)$ was calculated by multiplying the sum of the infarcted areas $\left(\mathrm{mm}^{2}\right)$ of the various sequential coronal sections by slice thickness $(1.5 \mathrm{~mm})(13,14)$. After estimating infarct volume by image analysis, the infarcted tissue in each slice was dissected and weighed as described above. To compensate for swelling, the following formula was applied: corrected infarct size $=$ infarct size $\mathrm{x}$ contralateral hemisphere size/ ipsilateral hemisphere size (15). The size of the infarct measured by the different methods was expressed as a percent of the affected (left) hemisphere.

\section{Experimental protocols}

Experiment 1. Rats were subjected to 2-h MCA occlusion, and infarct size was estimated $24 \mathrm{~h}$ later by both the balance method (weight, mg) and plethysmography (volume, $\left.\mathrm{mm}^{3} ; \mathrm{N}=41\right)$. In another group of animals $(\mathrm{N}=10)$, infarct size was first estimated by computerized image analysis (volume) and then by weighing (mg). The experiment using weighing and plethysmography was performed in duplicate to test the reproducibility of staining, dissecting and blotting the tissue, and weighing and plethysmography. This series of experiments was performed to evaluate whether the results obtained by weighing correlated with those obtained by 
plethysmography and digital image analysis.

Experiment 2. Rats were submitted to different durations of MCA occlusion (1.0, 1.5 and $2.0 \mathrm{~h} ; \mathrm{N}=7,8$ and 8 , respectively) and infarct size was estimated $24 \mathrm{~h}$ later by weighing. This experiment was designed to test the sensitivity of the weighing method to detect the effect of ischemia infarct size as a function of severity. It is well known that the extent of infarct increases consistently with duration of ischemia (16-18).

Experiment 3. This experiment was designed to test the sensitivity of the weighing method in detecting the effect of drugs on infarct size. Three series of experiments were carried out sequentially as follows: 1) the rats were given $50 \mathrm{mg} / \mathrm{kg}$ lamotrigine (LTG, 3,5-diamino-6-[2,3-dichlorophenyl]-1,2,4trizine; Lamictal ${ }^{\circledR}$ ) po, delivered $90 \mathrm{~min}$ (7) before a 2-h period of MCA occlusion $(\mathrm{N}=$ $30) ; 2)$ dipyrone $(100 \mathrm{mg} / \mathrm{kg}, p o)$, the first dose being delivered $30 \mathrm{~min}$ before ischemia, followed by two doses given 3 and $6 \mathrm{~h}$ later (19) $(\mathrm{N}=13)$; 3) LTG plus dipyrone as above $(\mathrm{N}=14)$. Saline $(0.9 \%)$ was given as a control for each experiment in a volume of $0.1 \mathrm{ml} / 100 \mathrm{~g}$ body weight $(\mathrm{N}=25,11$ and 10 , respectively).

\section{Statistical analysis}

Data were analyzed using StatGraphics Plus version 6.0 software. Pearson's correlation coefficient (r) was used to examine the relationship between the weight $(\mathrm{mg})$ and volume $\left(\mathrm{mm}^{3}\right)$ of the ischemic tissue (experiment 1). To evaluate change in infarct size as a function of ischemia duration, linear regression analysis was used (experiment 2). The effect of drug treatments on infarct size was compared by the Student $t$ test (experiment 3). The time course of core temperature in the different treatments (experiment 3) was analyzed by two-way MANOVA with one repeated level (17 time points) and one unrepeated level (groups); when a global main group effect was de- tected, the Newman-Keuls multiple range test was used to determine significant differences between groups, and the unpaired $t$ test was used to determine the time points at which the groups were different. When a main time effect was revealed, a withingroup, paired $t$-test was used to compare the basal temperature with that measured during and after ischemia. For this last comparison, the level of significance $(\alpha=0.05)$ was adjusted ( $\alpha=0.003)$ according to the Bonferroni procedure (20).

\section{Results}

In the present experiments, $27 \%$ of the animals died from hemorrhage shortly after the beginning of reperfusion. Hemorrhage was revealed by the presence of blood clots on the skull floor. An additional $11.4 \%$ of the animals were excluded because of the presence of non-lethal hemorrhage observed at the time of brain removal on the following day, and $2 \%$ were found dead on the morning of the following day but not checked for hemorrhage. Infarct location varied from a single striatal or cortical focus to a broad corticostriatal zone of necrosis enveloping almost the entire mediofrontal, dorsolateral portion of the affected hemisphere.

Figure 1 shows that infarct size as measured by the weight (mg, \% of affected hemisphere) of ischemic tissue correlated very well with its volume $\left(\mathrm{mm}^{3}, \%\right.$ of affected hemisphere) estimated by plethysmography or by computerized image analysis $(r=0.88$ 0.98; $\mathrm{P}<0.001-0.0001)$. These degrees of correlation were obtained by different pairs of observers, indicating that the experimental procedures involved in the weighing method are reproducible.

The sensitivity of the weighing method in detecting the effect of treatments on infarct size was established by the present results. Figure 2 (panel A) shows that the mass (mg) of the ischemic tissue increased linearly with the period of MCA occlusion (linear regres- 

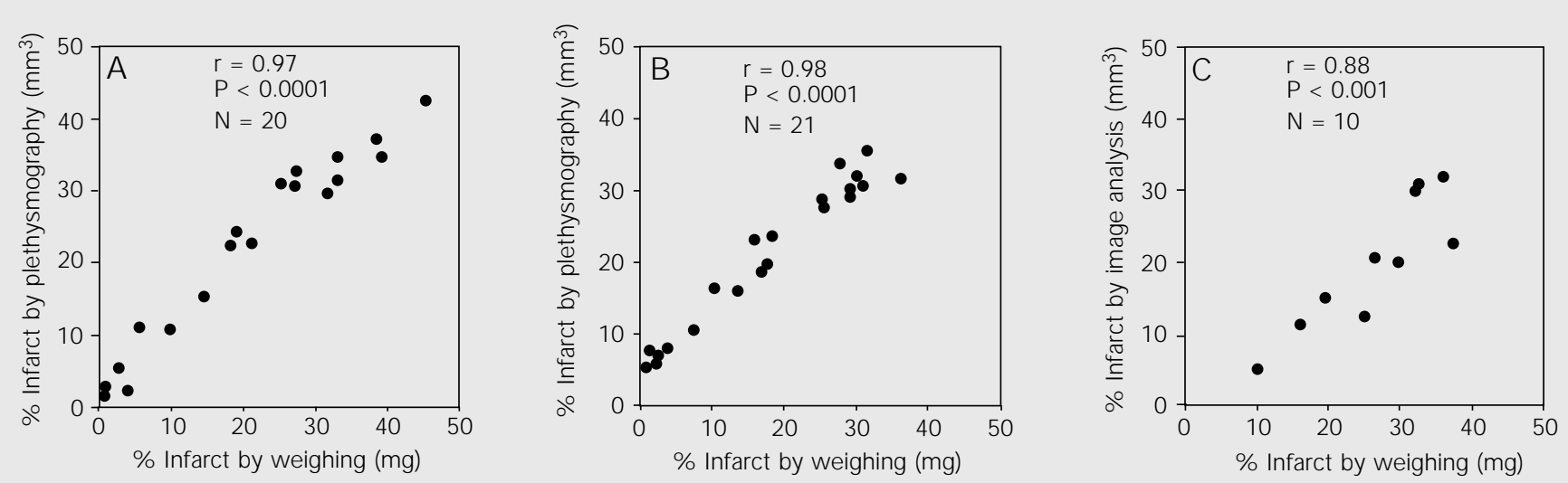

Figure 1. Relationship between infarct size estimated by mass and volume of ischemic brain tissue. Data are reported as percent infarct in relation to the affected hemisphere. Volume was estimated by plethysmography by two pairs of observers (panels $A$ and $B$ ) and by using an image analysis system (panel C). The data in panel A were obtained by S.J.Y. and L.K.C.M., the data in panel B by S.C.L.R. and C.J .L., and the data in panel C by L.K.C.M. and S.C.L.R. In each experiment, a given observer always performed the same set of experimental procedures. Each point represents data from an individual animal. In panel A, two animals had coincident infarct size (24.16\% and $24.21 \%)$.

sion: $\left.\mathrm{F}_{1,21}=11.36, \mathrm{P}<0.005\right)$. The effect of ischemia duration on infarct weight was similar to its effect on rectal temperature (Figure 2, panel B). Compared to basal values, core temperature increased abruptly until the end of each occlusion period (60, 90 or $120 \mathrm{~min})$ (Student $t$-test: $\mathrm{P}<0.001-0.0001 v s$ basal), dropping rapidly after the beginning of reperfusion when the animal was briefly reanesthetized with ether for retraction of the occluder. During reperfusion, however, the rectal temperature of rats subjected to 2-h ischemia was higher than the respective basal value $(\mathrm{P}<0.005)$, while in those subjected to 60- or 90-min occlusion, temperature returned to normothermic levels. A global analysis of variance considering the entire experimental period revealed a betweengroup effect $\left(\mathrm{F}_{2,280}=8.29, \mathrm{P}=0.0024\right.$; Newman-Keuls test: 120 vs 60 or $90 \mathrm{~min}, \mathrm{P}<$ $0.05)$. A point-to-point comparison revealed a significant difference at the end of the period of temperature recording (Student $t$ test: $\mathrm{P}<0.05)$. Hyperthermia was no longer present at the time of brain removal ( $24 \mathrm{~h})$, however, whatever the duration of ischemia (Student $t$-test: $\mathrm{P}>0.05 v s$ basal).

Figure 3 shows that the weighing method was also able to detect the effects of pharma-

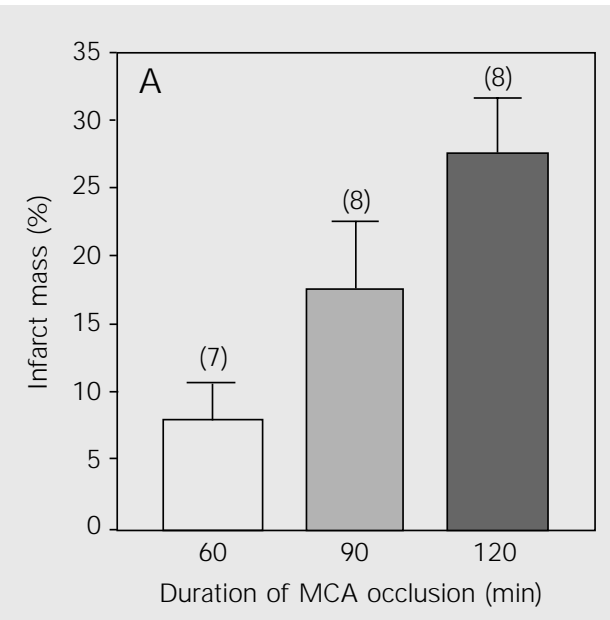

Figure 2. Influence of duration of medial cerebral artery (MCA) occlusion on infarct size (panel A) and core temperature (panel B). Data are the means \pm SEM and the number of animals studied in each group is given in parentheses. Infarct size was estimated by dissecting and weighing the ischemic tissue which is reported as a percent of the affected hemisphere. Infarct size increased with duration of ischemia $(P<$ 0.005). During ischemia, hyperthermia developed at the end of each occlusion period ( $\mathrm{P}<$ 0.001-0.0001 vs basal, rectangle). After reperfusion, rectal temperature was persistently higher in the group subjected to 2-h occlusion compared to the other groups. $* \mathrm{P}<0.005$ vs basal; $+\mathrm{P}<$ 0.05 , intergroup point-to-point comparisons; MANOVA/Newman-Keuls test: $P<0.05$. Adjusted $\alpha^{*}=0.003$. surg $=$ surgery; isc = ischemia. 
Figure 3. Effect of lamotrigine (LTG) alone or in combination with dipyrone (DIP) on ischemiainduced brain damage and hyperthermia. Data are the means \pm SEM and the number of animals studied in each group is given in parentheses. Compared to saline, LTG alone did not reduce brain infarct size (panel A, left column) or hyperthermia (panel B). Dipyrone alone also did not alter infarct size significantly (panel A, middle column), but reduced core temperature to the level of control in the reperfusion period (panel C; P > 0.05 dipyrone vs sham). Compared to saline, LTG + dipyrone reduced infarct size (panel $A$, right column, $+\mathrm{P}<0.005$ ) and prevented ischemia-induced hyperthermia (panel D; P < 0.05 LTG + DIP vs saline). The arrows in panels $C$ and $D$ indicate the time of oral dipyrone administration. $* P<$ 0.05-0.0001 compared to basal, rectangle); \#p < 0.05-0.0001, point-to-point comparison for saline vs sham (panel $C$ ) or saline vs LTG/dipyrone (panel D). Adjusted $\alpha^{*}=0.003$ for simultaneous, multiple t-test comparisons. surg = surgery; isch = ischemia. cological treatments on infarct size. In an initial experiment, pretreatment with LTG alone $(50 \mathrm{mg} / \mathrm{kg}, p o)$ did not reduce infarct size (panel A: $\mathrm{P}>0.05$, saline vs LTG; Student $t$-test). To establish that the failure of LTG to afford neuroprotection was due to a masking effect of ischemia-induced hyperthermia (panel B: $\mathrm{P}<0.0001$ vs basal) and not to a lack of method sensitivity, we ascertained (post hoc) whether reducing hyperthermia pharmacologically might improve the effect of LTG. The antipyretic effect of dipyrone on the hyperthermia induced by ischemia is illustrated in Figure 3 (panel C). A global main group effect was found (MANOVA, $\mathrm{F}_{2,563}=7.50, \mathrm{P}<0.0021$; Newman-Keuls test: $\mathrm{P}<0.01$, saline $v s$ sham). Dipyrone did not prevent hyperthermia during the intra-ischemic period. After reperfu-
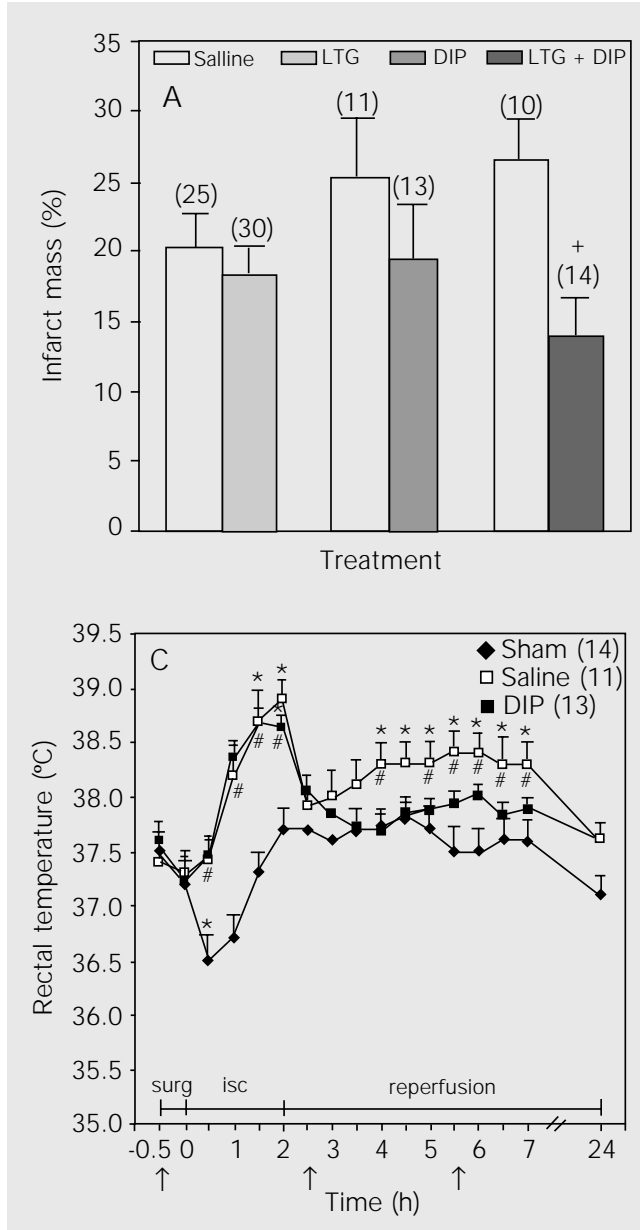

sion, the saline-treated group still exhibited elevated temperature (panel C: $\mathrm{P}<0.05 v s$ basal; Student $t$-test), which was reduced to normothermia in the dipyrone group (Newman-Keuls test: dipyrone $v s$ sham, $\mathrm{P}>0.05$ ); compared to the saline-treated group, however, the antipyretic effect of dipyrone was not statistically significant (Newman-Keuls test: $\mathrm{P}>0.05$ ). Dipyrone alone also did not significantly reduce infarct size (panel A, middle column). Considering the effect of dipyrone on core temperature during the reperfusion phase, we applied the same treatment protocol plus LTG in the subsequent experiment. Figure 3 shows the effect of LTG plus dipyrone on infarct size as measured by the weighing method. The LTG/ dipyrone combination reduced both infarct size (panel A, right column: $\mathrm{P}<0.005$; Stu-
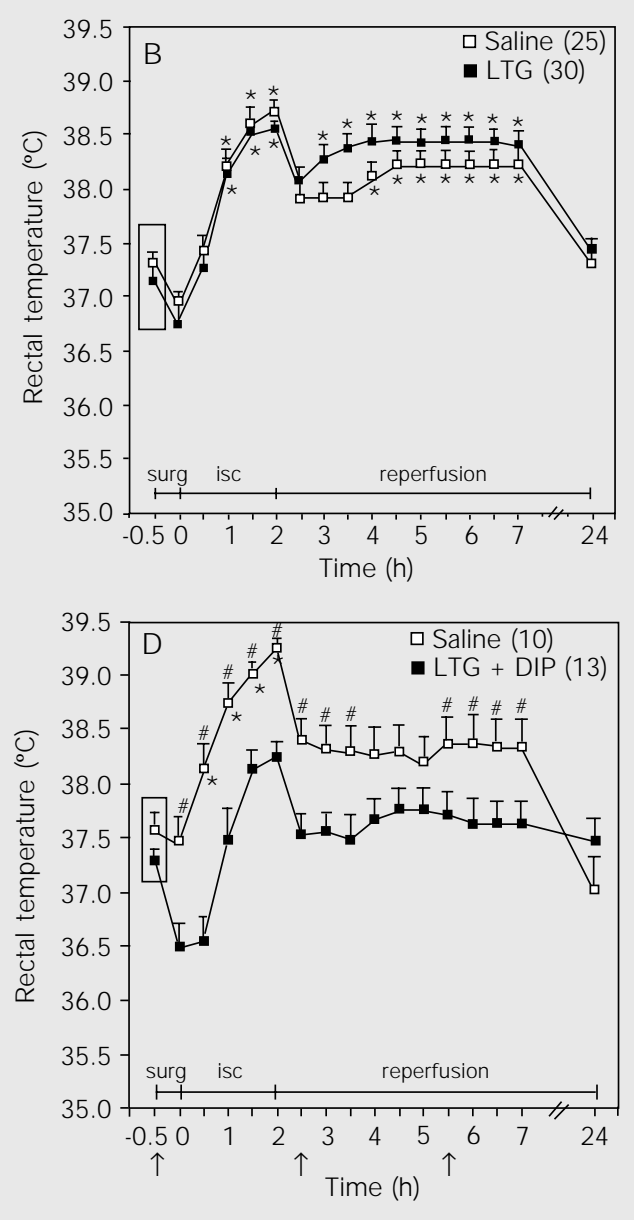
dent $t$-test) and the ischemia-induced hyperthermia throughout the period of temperature measurement (panelD: MANOVA, $\mathrm{F}_{1,337}$ $=7.45, \mathrm{P}<0.05 ; \mathrm{P}<0.005-0.0001 v s$ basal; $\mathrm{P}<0.05-0.0001$ intergroup point-to-point comparisons). The temperature recorded in the LTG/dipyrone group (panel D) was very similar to that in the sham group (panel B; MANOVA, $F_{1,402}=0.80, P=0.39$ ), suggesting that together dipyrone and LTG exerted an antipyretic action that may have facilitated the neuroprotective effect of LTG. The weighing method was sufficiently sensitive to distinguish between the effects of such treatments.

\section{Discussion}

We determined if the method involving dissecting and weighing ischemic tissue might serve as a sensitive, reproducible and low-cost alternative to quantify the extent of brain infarct in rodent models of focal cerebral ischemia.

A well-recognized aspect of infarcts induced in this animal model concerns the wide variability in infarct size. Such variability occurs not only between studies by different investigators, but also within groups of animals studied by the same individual(s) (21). Apart from individual variability in cerebrovascular anatomy, several model-inherent complications apparently contribute to variability in infarct size (22). The high mortality rate reported in the present study was mainly due to hemorrhage caused by introduction or retraction of the occluder. A similar mortality rate $(33 \%)$ has been reported $48 \mathrm{~h}$ after transient MCA occlusion in rats (23). In our procedures, Ethicon nylon monofilament induces considerably less hemorrhage when compared to other types of nylon monofilament (for example, 4-0 Superlon, Cirumédica Ltda., Cotias, SP, Brazil).

The weighing method was apparently used for the first time by Hoff et al. (12) to quantify the effect of pentobarbital on infarct size in cats. Since Hoff's report does not provide methodological details of the procedures adopted to dissect and weigh the ischemic brain tissue, the dissecting and weighing protocols used in the present study cannot be compared to those of Hoff et al. (12). Possibly the weighing method has not been used since its description in 1982 because of the lack of methodological details. Since then, however, other techniques have been developed to estimate infarct size, including comparisons of single representative coronal sections (24), comparison and extrapolation of TTC- and hematoxylin/ eosin-stained coronal sections after photographic projection and outlining infarcted and normal areas (3), and planimetry of multiple sections after projecting the infarcted area on scale diagrams $(25,26)$. Although these methods provide excellent reproducibility, they are tedious and difficult to accomplish in an accurate and objective manner (10). Most recently, semiautomatic (10) and automated (11) methods requiring sophisticated image analysis systems have been developed to provide quantitative infarct assessment. Variants of these methods are known $(7,27)$, but are still costly enough to limit or hinder work by many laboratories.

As used by us and detailed here, the dissecting and weighing method is simple and easy to carry out once the experimental procedures are standardized and become a routine in practice. The TTC histochemical staining step constitutes a critical point for the success of the method. The high-contrast colors between the infarcted (white) and normal (deep red) tissues make the dissection process easy, without the need for a dissecting microscope. Provided a good standard of staining is attained, a reliable dissection is guaranteed. However, the dissection process requires care, particularly in sections where the infarct does not cross the coronal section completely from one surface to the other (usually the first and last sections 
containing the ischemic tissue from the frontal to the occipital pole, respectively). Although infarct size may be small in many animals, the ischemic necrotic tissue is easily discernible and may become partially separated from normal tissue. This spontaneous detachment may become more evident as the duration of reperfusion increases from 24 to $48 \mathrm{~h}$, an interval often used to evaluate the acute, neuroprotective effects of drugs. The degree of difficulty in dissecting the ischemic tissue away from the intact tissue is similar to that of outlining the border when using a semiautomatic computerassisted, image analysis system. Compared to the image analysis procedure, the dissecting and weighing method may be more precise in that the infarct profile in different cross-sections at different depths of section thickness will not be uniform, at least in some sections. The estimation of infarct size (volume, $\mathrm{mm}^{3}$ ) by digital image analysis relies on establishing the ischemic area $\left(\mathrm{mm}^{2}\right)$, outlined on one of the section surfaces, while the dissection method evaluates the entire infarcted tissue distributed across all levels of the coronal sections. Thus, the dissection and weighing method may provide a more practical and accurate estimate of the ischemic tissue mass. This may explain the weaker correlation between weight and volume estimated by image analysis $(\mathrm{r}=$ 0.88 ) compared to that between weight and volume estimated by plethysmography ( $\mathrm{r}=$ 0.98), although fewer rats were used in the plethysmography group. Both plethysmography and the weighing method require the same dissection procedure.

Since the border between the ischemic and normal tissue usually appears pink in color and is embedded in normal tissue, dissection can be performed using the deep red color of the intact tissue as a reference. Whether the pink-colored tissue may evolve to complete necrosis or recover after a period of reperfusion longer than $24 \mathrm{~h}$ is not known. Although the ischemic (necrotic) tissue undergoes gradual degradation over time to finally form a necrotic cavity $(13,28)$, the weighing method is not limited to the acute period of ischemia/reperfusion. It is equally applicable when the aim is to measure the infarct size beyond 24 to $48 \mathrm{~h}$ of reperfusion. In this case, measurement of infarct size can be estimated on the basis of the remaining intact tissue compared to the contralateral hemisphere.

The present results suggest that the method of dissection and weighing is a reliable alternative for measuring the extent of brain infarct in animal models. The reliability of the method was evaluated first by demonstrating that the mass $(\mathrm{mg})$ of the ischemic tissue closely correlated with its volume $\left(\mathrm{mm}^{3}\right)$ measured by plethysmography or using an image analysis system. However, this does not establish the accuracy of the present method, since the sensitivity of computer-assisted image analysis systems has apparently not been examined thus far (7). Although the plethysmography technique apparently has not been used to measure brain infarct, it is a well-characterized and extensively used method to quantify the volume of paw edema experimentally induced in rats (29). The technique provides a precise and efficient method when using a hydroplethysmometer, particularly if two platinum electrodes immersed in an electrolyte are employed to measure changes in fluid level (30). Recently, the use of mass to express the volume of fluid displaced by an object has been reported as a simple and accurate alternative method compared to the technique of hydroplethysmography using a platinum electrode (30) which, in turn, is still an expensive device. The use of hydroplethysmography as a comparative method in the present study raises the question of whether the volume of ischemic or normal tissue is accurately measured when tissue is immersed in the plethysmometer electrolyte solution owing to absorption or loss of water. Osmotic water movement is unlikely since the tissue was 
previously washed twice in the same electrolyte solution ( $0.07 \%$ saline). The close correlation demonstrated between mass and volume of ischemic tissue, as well as the high degree of interobserver reproducibility (Figure 1) show that the volume did not change when the tissue was transferred from the balance to the plethysmometer electrolyte solution.

The weighing method was also capable of detecting changes in infarct size after different treatments. It is well known that the duration of ischemia is a determining factor in the severity of brain damage $(15,16,18)$. Thus, the duration of MCA occlusion should represent a consistent and appropriate variable to estimate a corresponding increase in infarct size which, in turn, is useful to test the sensitivity of the method. This was confirmed by a strong and consistent alteration in infarct size as a function of ischemia duration (Figure 2). Although it is well known that ischemia may cause fever, the manner by which its duration affected rectal temperature in the present study deserves further analysis. During occlusion, the degree and time course of ischemia-induced hyperthermia were similar in the various animal groups. When the occluder filament was removed the elevated temperature dropped abruptly, probably due to anesthesia. Despite this, however, hyperthermia was still present during reperfusion in the group subjected to 120-min ischemia, but not in those submitted to 60- or 90-min ischemia. However, there was no relationship between infarct size and rectal temperature measured at different points during the ischemic and reperfusion periods (data not shown).

The weighing method was also able to detect the effect of drugs on infarct size. Currently, there is no standard neuroprotective agent available which could be used as a positive control. We chose LTG because it has been used recently to validate a modified image analysis method for quantification of brain infarct (7). LTG is known for its neuroprotec- tive properties when given prior to global (3133 ) or focal cerebral ischemia in animal models $(7,34)$. It is rapidly absorbed by the enteral route (33) and easily available as an antiepileptic agent. The weighing method can distinguish the masking effect of ischemia-induced hyperthermia on the neuroprotective efficacy of LTG. It is well known that moderate increases in body temperature not only aggravate the outcome of brain ischemia $(35,36)$ but can mask or annul the neuroprotective effect of drugs (37). The degree of body hyperthermia induced by 2 -h ischemia in the present study is consistent with previous reports (35). While dipyrone alone reduced hyperthermia during the period of reperfusion alone (Figure 3, panel C), the combination of dipyrone and LTG prevented hyperthermia during both the ischemic and reperfusion periods (panel D). Whether these drugs interact to produce a synergistic, antipyretic effect is not known. Such an antipyretic effect may have revealed the neuroprotective effect of LTG. This observation agrees with previous studies showing that LTG provides neuroprotection in rats with MCA occlusion maintained normothermic by physical means $(7,34)$. Although dipyrone alone did not induce a significant reduction in infarct size, a possible synergistic, neuroprotective effect of LTG and dipyrone cannot be ruled out. Dipyrone has been shown to reduce convulsions in different animal models of epilepsy, possibly indicating an antiexcitotoxic property of dipyrone (38). Also, dipyrone has been shown to reduce delayed neuronal cell death after transient global cerebral ischemia. This effect, however, has been attributed to the antipyretic action of dipyrone (19).

The present study demonstrates that the extent of cerebral infarct induced experimentally in animals can be reliably quantitated by dissecting and weighing the ischemic tissue after TTC staining. If an acute reduction in infarct size is taken to be indicative of neuroprotection, the weighing method exhibits the sensitivity and reproducibility nec- 
essary to detect a neuroprotective effect of certain drugs. The main advantage of the technique over computer-assisted image analysis lies in its low cost, with no need for expensive equipment. Skill in staining and dissecting the ischemic tissue from the normal tissue is necessary, and an analytical balance is required. The weighing method may thus constitute a viable alternative for many laboratories.

\section{Acknowledgments}

The authors gratefully acknowledge Dr. Ciomar Bersani Amado and Jailson Araujo Dantas (Departamento de Farmácia e Farmacologia) for technical assistance in the use of the plethysmometer, and José António de Souza (Departamento de Ciências Morfofisiológicas) for assistance with the computerized image analysis system. We also thank Marcos A. Trombelli for daily animal care.

\section{References}

1. Altaman FP (1976). Tetrazolium salts and formazans. Progress in Histochemistry and Cytochemistry, 9: 1-56.

2. Sharkey J \& Butcher SP (1984). Immunophilins mediate the neuroprotective effects of FK506 in focal cerebral ischaemia. Nature, 371: 336-339.

3. Bederson J B, Pitts LH, Tsuji M, Nishimura MC, Davis RL \& Bartkowski H (1986). Rat middle cerebral artery occlusion: evaluation of the model and development of a neurologic examination. Stroke, 17: 472-476.

4. Longa EZ, Weinstein PR, Carlson S \& Cummins R (1989). Reversible middle cerebral artery occlusion without craniotomy in rats. Stroke, 20: 84-91.

5. Bartus RT, Baker KL, Heiser AD, Sawyer SD, Dean RL, Elliott PJ \& Straub J A (1994). Postischemic administration of AK275, a calpain inhibitor, provides substantial protection against focal ischemic brain damage. J oumal of Cerebral Blood Flow and Metabolism, 14: 537544.

6. Maier CM, Ahern K-VB, Cheng ML, Lee J E, Yenari MA \& Steinberg GK (1998). Optimal depth and duration of mild hypothermia in a focal model of transient cerebral ischemia. Effects on neurological outcome, infarct size, apoptosis, and inflammation. Stroke, 29: 21712180.

7. Yang Y, Shuaib A \& Li Q (1998). Quantification of infarct size on focal cerebral ischemia model of rats using a simple and economical method. J ournal of Neuroscience Methods, 84: 9-16.

8. Yoshimoto T\& Siesjö BK (1999). Posttreatment with the immunosuppressant cyclosporin A in transient focal ischemia. Brain Research, 839: 283-291.

9. Traystman RJ , Klaus J A, DeVries AC, Shaivitz AB \& Hum PD (2001). Anticonvulsant lamotrigine administered on reperfusion fails to improve experimental stroke outcomes. Stroke, 32: 783-787.

10. Swanson RA, Morton MT, Tsao-Wu G, Savalos RA, Davidson C \& Sharp FR (1990). A semiautomated method for measuring brain infarct volume. J ournal of Cerebral Blood Flow and Metabolism, 10: 290-293.

11. Goldlust EJ , Paczynski RP, He YY, Hsu CY \& Goldberg MP (1996). Automated measurement of infarct size with scanned images of triphenyltetrazolium chloride-stained rat brains. Stroke, 27: 16571662.

12. Hoff J T, Nishimura $M \&$ Newfield $P$ (1982). Pentobarbital protection from cerebral infarction without suppression of edema. Stroke, 13: 623-628.

13. Hong J T, Ryu SR, Kim HJ , Lee J K, Lee SH, Kim DB, Yun YP, Ryu J H,
Lee BM \& Kim PY (2000). Neuroprotective effect of green tea extract in experimental ischemia-reperfusion brain injury. Brain Research Bulletin, 53: 743-749.

14. Ünal I, Gursoy-Özdemir Y, Bolay $H$, Söylemezoglu F, Saribas $O \&$ Dalkara T (2001). Chronic daily administration of selegiline and Egb 761 increases brain's resistance to ischemia in mice. Brain Research, 917: 174-181.

15. Chang ML, Yang J, Kem S, Klaidman L, Sugawara T, Chan PH \& Adams J D (2002). Nicotinamide and ketamine reduce infarct volume and DNA fragmentation in rats after brain ischemia and reperfusion. Neuroscience Letters, 322: 137-140.

16. Garcia J H, Yoshida Y, Chen H, Li Y, Zhang ZG, Lian J, Chen S \& Chopp M (1993) Progression from ischemic injury to infarct following middle cerebral artery occlusion in the rat. American J oumal of Pathology, 142: 623-635.

17. Siesjö BK, Katsura K-I, Zhao Q, Folbergrová J , Pahlmark K, Siesjö P \& Smith M-L (1995). Mechanisms of secondary brain damage in global and focal ischemia: a speculative synthesis. Journal of Neurotrauma, 12: 943-955

18. Nagahiro S, Uno M, Sato K, Goto S, Morioka M \& Ushio Y (1998). Pathophysiology and treatment of cerebral ischemia. J ournal of Medical Investigation, 45: 57-70.

19. Coimbra C, Boris-Moller F, Drake M \& Wieloch T (1996). Diminished neuronal damage in the rat brain by late treatment with the antipyretic drug dipyrone or cooling following cerebral ischemia. Acta Neuropathologica, 92: 447-453.

20. Krauth J (1988). Distribution-Free Statistics: An Application-Oriented Approach. Vol. 2. Elsevier, Amsterdam, The Netherlands.

21. Avendaño C, Roda J M, Carceller F \& Díez-Tejedor E (1995). Morphometric study of focal cerebral ischemia in rats: a stereological evaluation. Brain Research, 673: 83-92.

22. Schmid-Elsaesser $R$, Zausinger $S$, Hungerhuber $E$, Baethmann $A$ \& Reulen H-J (1998). A critical reevaluation of the intraluminal thread model of focal cerebral ischemia: Evidence of inadvertent premature reperfusion and subarachnoid hemorrhage in rats by laserdoppler flowmetry. Stroke, 29: 2162-2170.

23. Aspey BS, Cohen S, Patel Y, Terruli M \& Harrison MJ G (1998), Middle cerebral artery occlusion in the rat: consistent protocol for a model of stroke. Neuropathology and Applied Neurobiology, 24: 487-497.

24. Coyle P, Odenheimer DJ \& Sing CF (1984). Cerebral infarction after middle cerebral artery occlusion in the rat by intraluminal suture. Stroke, 15: 711-716. 
25. Lundy EF, Solik BS, Frank RS, Lacy PS, Combs DJ , Zelenock GB \& D'Alecy LG (1986). Morphometric evaluation of brain infarcts in rats and gerbils. J ournal of Pharmacological Methods, 16: 201-214.

26. Osborne KA, Shigeno T, Balarsky AM, Ford I, McCulloch J , Teasdale GM \& Graham DI (1987). Quantitative assessment of early brain damage in a rat model of focal cerebral ischaemia. J ournal of Neurology, Neurosurgery and Psychiatry, 50: 402-410.

27. Zhao W, Belayev L \& Ginsberg M (1997). Transient middle cerebral artery occlusion by intraluminal suture: II. Neurological deficits, and pixels-based correlation of histopathology with local blood flow and glucose utilization. J oumal of Cerebral Blood Flow and Metabolism, 17: 1281-1290.

28. Nishino H, Czurkó A, Fukuda A, Hashitani T, Hida H, Karadi Z \& Lénard L (1994). Pathophysiological process after transient ischemia of the middle cerebral artery in the rat. Brain Research Bulletin, 35: 51-56.

29. Milanino R (1988). Synthesis and anti-inflammatoy effect of some bis (2-benzimidazolyl) thiolethers and their copper (II) chelates, orally administered to rats. European J oumal of Medicinal Chemistry, 23: 217-224.

30. Fereidoni M, Ahmadiani A, Semnanian S \& J avan M (2000) An accurate and simple method for measurement of paw edema. J ournal of Pharmacological and Toxicological Methods, 43: 11-14.

31. Wiard RP, Dickerson MC, Beek O, Norton R \& Cooper BR (1995).
Neuroprotective properties of the novel antiepileptic lamotrigine in a gerbil model of global cerebral ischemia. Stroke, 26: 466-472.

32. Shuaib A, Mahmood RH, Wishart T, Kanthan R, Murabit MA, Ijaz S, Miyashita $H$ \& Howlett W (1995). Neuroprotective effects of lamotrigine in global ischemia in gerbils. A histological, in vivo microdialysis and behavioral study. Brain Research, 702: 199-206.

33. Crumrine RC, Bergstrand K, Cooper AT, Faison WL \& Cooper BR (1997). Lamotrigine protects hippocampal CA1 neurons from ischemic damage after cardiac arrest. Stroke, 28: 2230-2237.

34. Smith SE \& Meldrum BS (1995). Cerebroprotective effect of lamotrigine after focal ischemia in rats. Stroke, 26: 117-122.

35. Zhao Q, Memezawa H, Smith ML \& Siesjo BK (1994). Hyperthemia complicates middle cerebral artery occlusion induced by an intraluminal filament. Brain Research, 649: 253-259.

36. Ginsberg MD \& Busto R (1998). Combating hyperthermia in acute stroke: A significant clinical concern. Stroke, 29: 529-534.

37. Memezawa H, Zaho Q, Smith ML \& Siesjo BK (1995). Hyperthermia nullifies the ameliorating effect of dizocilpine maleate (MK801) in focal cerebral ischemia. Brain Research, 670: 48-52.

38. Doretto MC, Garcia-Cairasco N, Pimenta NJ G, Souza DA \& Tatsuo MAKF (1998). Dipyrone, a novel anticonvulsant agent? Insights from three experimental epilepsy models. NeuroReport, 9: 24152421. 